\title{
Use of microcomputer to help teach pathology
}

\author{
M C ANDERSON, D W FORD* \\ Departments of Histopathology and *Bioengineering, St Mary's Hospital Medical School, London
}

SUMMARY A BBC microcomputer was incorporated into a closed circuit television system while it ${ }^{\circ}$ was being used to show microscope slides. The computer generates screens of text that complement $\vec{\circ}$ the demonstration of the microscopy. An additional feature is the capacity of the system to produce $\overrightarrow{-}$ $35 \mathrm{~mm}$ transparencies.

Many departments of pathology use closed circuit television (CCTV) to show histological material. While teaching is in progress it is often useful to be able to show text to the audience, perhaps to present a short case summary relating to the slides being shown, or to illustate a point raised by the slides. For example, if a section of an ovarian tumour is being shown to an audience of medical students it helps to show a classification of ovarian tumours to remind them where the tumour in question fits into that classification. A clinical summary for a case presentation could be shown by transparencies on an overhead projector and the classification would most probably entail using $35 \mathrm{~mm}$ transparencies and a slide projector. Switching attention from one visual medium, the television screen, to a second, or even a third medium, the overhead projector or the slide projector, requires an adjustment of vision for the audience and inevitably interferes with concentration. The system described here overcomes this disadvantage of conventional systems and in addition has other advantages over both other methods of showing text. We do not claim this as an original idea; it would be surprising if many other users of CCTV had not developed similar, perhaps better, systems. The equipment described here, however, was extremely useful in the context of this department; and it is also simple to use and set up.

The basis of the system is a BBC microcomputer with disc drive for which the lettering produced in the teletext mode (mode 7) is used. With this mode on the computer, lines of script can be written on the screen in clear letters in two sizes and seven colours. Crude graphics can also be generated, but for our purposes these were confined to text separating lines. Although it is possible to write the required program in Basic,

Accepted for publication 8 May 1986 this is very time consuming, and it is much quicker and easier to use a commercially available software $\vec{O}$ package; such as "Teletext Editor" by Beebugsoft, $\overrightarrow{\vec{A}}$ which has been used and found to be entirely satis- $\vec{t}$ factory. This program enables the operator to type 으 the text on to the screen as rapidly as a word pro- $\triangle$ cessor would permit. The software program then has $O$ the facility to save screens on to disc and incorporate them into any other Basic program. Figs 1 and $2 \frac{0}{8}$ illustrate the phases of the program.

In the second half of the skeleton program a pros $\overrightarrow{0}$ cedure is defined for each key specified in the first hasf of the program. In practice, this "skeleton" program is loaded into the computer, and the lines of program written by the teletext editor for each screen are inserted into this main program using the *EXEC

Fig 1 First phase of skeleton computer program

\begin{tabular}{l}
\hline Skeleton computer program: \\
10 REM "Skeleton" \\
20 MODE 7 \\
30 VDU12,23;8202;0;0;0; \\
$40 \mathrm{~K} \%=$ GET:CLS \\
50 IF K $\%=49 *$ THEN PROCONE \\
60 IF K $\%=50$ THEN PROCTWO \\
70 IF K $\%=51$ THEN PROCTHREE \\
80 IF K $\%=52$ THEN PROCFOUR \\
90 IF K $\%=53$ THEN PROCFIVE \\
100 IF K $\%=54$ THEN PROCSIX
\end{tabular}

*The numbers are the ASCII codes for the keys specified in the PROC.... phrase.

Fig 2 Second phase of skeleton computer program

\begin{tabular}{l}
\hline 410 GOTO 40 \\
420 END \\
450 DEFPROCONE \\
690 ENDPROC \\
700 DEFPROCTWO \\
1000 ENDPROC \\
1010 DEFPROCTHREE \\
1300 ENDPROC
\end{tabular}

410 GOTO 40

690 ENDPROC

700 DEFPROCTWO

1010 DEFPROC

1300 ENDPROC 


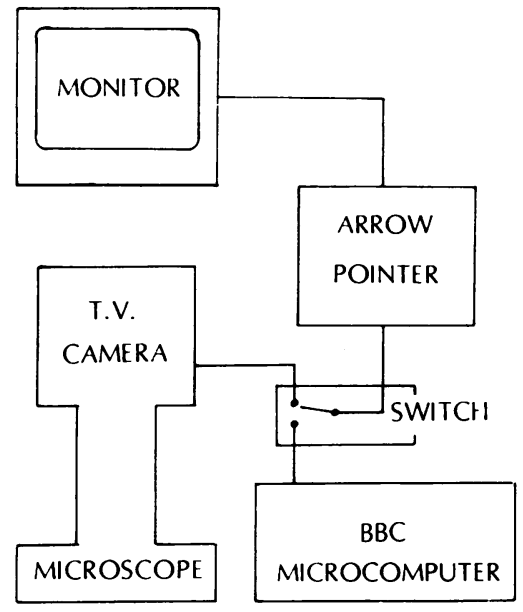

Fig 3 Connections that are made between CCTV camera, computer, and monitor.

command. When the program is chained, a touch of the appropriate key will bring up the required text screen; at the touch of another key a different screen is instantly displayed. We used a different "skeleton" program for each main topic and renamed them appropriately, such as "vulva," "cervix," "endometrium" and "ovary." To save time during the presentations each of these programs is called using a function key, which is programmed by loading a program such as this as a preliminary:

\section{0 *KEY $0 \mathrm{CH}$. "VULVA" $\mathrm{M}$}

20 *KEY $1 \mathrm{CH}$. "CERVIX" $M$

and so on.

Pressing function key 1 will load the "cervix" program ready for use. This preliminary program can be incorporated into a "!BOOT" program so that it is loaded as soon as the computer is booted. For use the computer is switched on, "shift" and "break" are pressed, then the required function key, followed by the appropriate keyboard key to bring up the required screen of text.

Using such a program, a large number of screens of text can be stored on one floppy disc, and any screen can be recalled by pressing only one or two keys on the computer during the teaching session. As a rough guide to the work entailed in preparation the author is able to create between eight and 12 screens an hour, depending on their complexity.

The hardware requirements are the same as those for a BBC microcomputer with disc drive, and for these purposes, two modifications are also required. Information is passed from the computer to the television monitors through video leads with a nominal impedance of $75 \mathrm{Ohms}$, so that the video output from the computer is used. In the $\mathrm{BBC}$ microcomputer the video output is black and white only and a modification is needed to convert this video output to colour.

\section{MODIFICATIONS TO THE BBC MICROCOMPUTER Part 1}

Conversion from black and white to colour video output requires a 470 picofarad ceramic capacitor connected between the emitter of transistor Q9 (a BC239 which is found between IC45 and the tin case of the ultra high frequency modulator) and the base of transistor Q7 (a BC309 which is found next to the empty IC97), which will provide a colour video output.

\section{Part 2}

Switching and interconnections of video signals entail a single pole, double throw switch with gold plated contacts and connections, and a surface mounting 75 $\mathrm{Ohm}$ BNC socket. The wire from the circuit board is connected to one of the switched contacts on the switch. The video output is then connected to the pole of the switch, which now becomes the system output. The new BNC socket is fixed to the back plate and connected to the remaining contact on the switch; this can now be placed as desired. All connections should be made with screened cable, keeping unscreened lengths as short as possible. The new BNC socket is the input from the video camera, the original BNC socket is the output to the monitors, and the switch selects the function to be displayed.

The video signal from the camera is fed into the back of the computer through an additional video input plug and then to a switch that can be operated from the back plate of the computer. The video output from the computer is then taken to an arrow pointer, so that the pointer can be used on the picture from the camera and also on the text from the computer (fig 3).

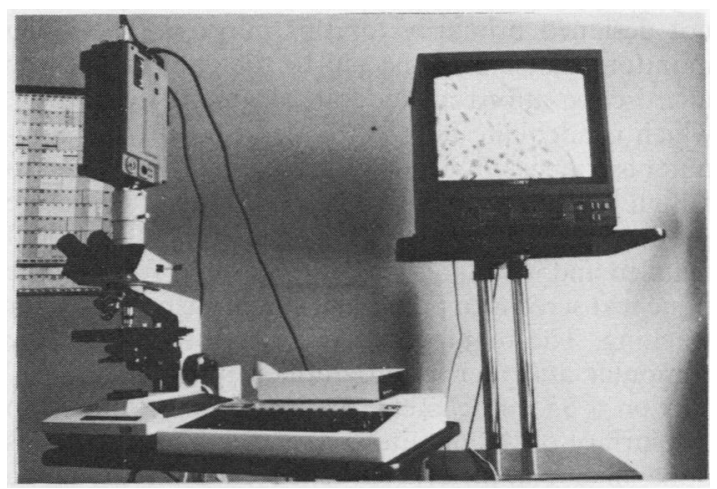

Fig 4 System in use, showing histology slide on monitor screen. 


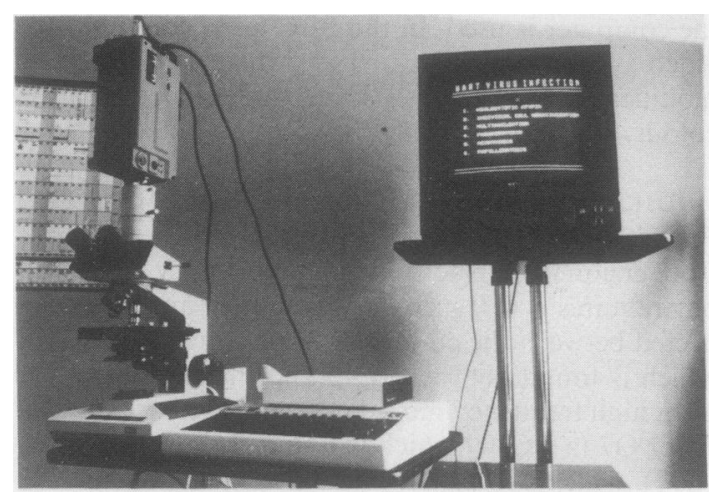

Fig 5 When switch is moved, list of diagnostic features relevant to slide appears on screen.

The use of the system requires two stages: the preparation of the text material and its presentation. The preparation has to be done beforehand as the text screens cannot be written while teaching is in progress. During the teaching period, the computer, video camera, microscope, and monitors remain connected and any of the assortment of text screens can instantly be recalled on to the monitors by flicking the switch on the back of the computer and keying the appropriate command on the computer. A major advantage of this system over diazo slides in a slide projector is that the text screens may be recalled in any order and the same screen can be called up on many occasions without the need to run through other slides that are not required, as would be necessary on a slide projector. Figs 4 and 5 show the system in use.

The enthusiastic user will soon realise that the system can be used not just for text screens but also for a whole range of charts and diagrams that can be written on other programs and recalled by the computer. This requires slightly more familiarity with computers but advanced knowledge is not required.

The apparatus has a further use, although it was not designed primarily for this purpose. The video monitors that are connected to the camera and the microscope afford a moderate degree of resolution, which is adequate for the use described. If the computer is connected through its red/green/blue outlet to a high resolution monitor, however, then the picture produced on the screen becomes very sharp and detailed and is suitable for photography. We used the same text screens to prepare $35 \mathrm{~mm}$ transparencies for teaching. The required screen is called up from the computer and then photographed using a "macro" lens on a $35 \mathrm{~mm}$ camera. To avoid reflections from the surface of the monitor screen the photography is done in a completely darkened room, although a hood could be constructed to keep out unwanted light. It is imperative to ensure that the camera is cor- rectly aligned in front of the screen; the slightest deviation will cause curvature of lines. If the monitor is of sufficiently high quality and the camera is directly in front of its centre no distortion will be discernible on the photograph.

Exposure can be determined by trial and error, or by using a spot meter, the reading being taken from the lettering rather than the background. Use of the camera's own exposure meter will result in overexposure as it tends to integrate to grey. As a rough guide, a shutter speed of half a second at 14.5 with a 64 ISO film should be about right, although exposure will vary depending on the brightness of the monitor. A shutter speed of $1 / 15$ second or longer is essential, otherwise the whole screen of the monitor may not appear on the finished transparency. The camera must therefore be on a tripod. Fig 6 shows the results of this technique, which are very acceptable. At first, one is tempted to use a variety of colours, graphics, and backgrounds on the screens, but experience has shown that simple combinations are the most effective. It is advisable to keep to the same colour combinations for slides prepared for the same lecture.

Many hospitals and medical schools have excellent departments of medical illustration, in which artwork is professionally prepared and photographed. The technique described here is not a substitute for these: it cannot match the quality of the final slide produceg in these departments. Most other hospitals, however have only a photographic department to which the camera ready work has to be submitted for preparation of diazo or black and white slides. In these circumstances the method described here has the advantages of ease and speed of preparation with the possibility of a final slide being produced in a very short time, if rapid film processing is used.

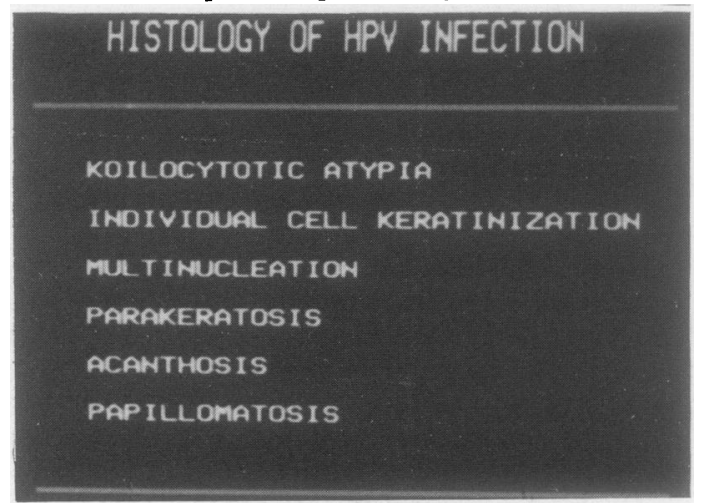

Fig 6 Monochrome copy of text screen as it appears on monitor.

Requests for reprints to: MC Anderson, Samaritan Hospital $\stackrel{\infty}{+}$ for Women, Marylebone Road, London NW1 5YE, 7 England. 